\title{
Stochastic analysis of an ecosystem of two competing species
}

\author{
G Q CAI and Y K LIN \\ Center for Applied Stochastics Research, Florida Atlantic University, \\ Boca Raton, FL 33431, USA \\ e-mail: caig@fau.edu
}

\begin{abstract}
A model describing two competing species of grass and woody vegetation in a semi-arid savanna grazing system is generalized to include a bounded stochastic variation in the cattle-stocking rate, and the system behaviour is investigated using a Monte Carlo-type simulation technique. Some key characteristics of the stochastic system are found to be different from the corresponding deterministic system without the stochastic variation. A single stable state in the deterministic system is diffused into a region of stable states, and a separatrix dividing the two attraction zones no longer exists. Effects of the intensity and the mean frequency of the stochastic variation of the cattle-stocking rate on the system behaviour are investigated.
\end{abstract}

Keywords. Ecosystem; competing species; stochastic model; Monte Carlo simulation.

\section{Introduction}

In its natural state, a semi-arid savanna has a predominant grass cover, with scattered trees and shrubs. Such a natural state is found in the south-western USA, and in Africa, India and Australia. Sustainability, stability and resilience of such a state have generated considerable interest among ecologists. Early studies of the dynamics of such systems were presented by Ludwig et al (1978) and Walker et al (1981). More recently, Ludwig et al (1997) presented a comprehensive investigation based on a modified version of the well-known model of Lotka (1925) and Volterra (1926) as follows,

$$
\begin{aligned}
& \dot{g}=r_{g} g\left(1-s-c_{g g} g-c_{w g} w\right), \\
& \dot{w}=r_{w}\left[a+w\left(1-c_{g w} g-c_{w w} w\right)\right],
\end{aligned}
$$

where $g$ and $w$ are the densities of the grass and woody vegetation respectively, $r_{g}$ and $r_{w}$ are their growth rates, $s$ is the cattle-stocking rate, $c_{w g}$ and $c_{g w}$ are the interspecies-competition coefficients, $c_{g g}$ and $c_{w w}$ are the intraspecies-competition coefficients, and $a$ is a source term for the woody vegetation. Model (1) differs from the original Lotka-Volterra model by

This paper is dedicated to Prof R N Iyengar of the Indian Institute of Science on the occasion of his formal retirement. 
adding the intraspecies competition terms $-c_{g g} g^{2}$ and $-c_{w w} w^{2}$, a source term to the woody vegetation, and a cattle-stocking term. Equation set (1) is deterministic; namely, all parameters in the set are assumed to be precisely known. The present paper is aimed at shedding some light on the profound effects of the variability of the system parameters with time.

\section{Some fundamental properties of the deterministic system}

To compare with the results to be presented of the stochastic analysis, a brief discussion of some basic properties of the deterministic system (1) is required. The equilibrium points of system (1) can be found by letting

$$
\begin{aligned}
& g\left(1-s-c_{g g} g-c_{w g} w\right)=0, \\
& a+w\left(1-c_{g w} g-c_{w w} w\right)=0 .
\end{aligned}
$$

Denoting

$$
A=c_{w g} c_{g w}-c_{g g} c_{w w}, \quad B=c_{g w}(1-s)-c_{g g}, \quad C=a c_{g g},
$$

the following three equilibrium centres exist if $B^{2}-4 A C>0$.

Unstable centre $\mathbf{S}$ :

$$
w=(1 / 2 A)\left[B+\left(B^{2}-4 A C\right)^{1 / 2}\right], g=\left(1-s-c_{w g} w\right) / c_{g g} .
$$

Asymptotic stable centre $\mathbf{W}$ :

$$
w=\left(1 / 2 c_{w w}\right)\left[1+\left(1+4 a c_{w w}\right)^{1 / 2}\right], g=0
$$

Asymptotic stable centre G:

$$
w=(1 / 2 A)\left[B-\left(B^{2}-4 A C\right)^{1 / 2}\right], g=\left(1-s-c_{w g} w\right) / c_{g g} .
$$

On the other hand, if $B^{2}-4 A C=0$, then system (1) has an unstable equilibrium centre $\mathbf{S}$, and an asymptotic stable equilibrium centre $\mathbf{W}$. Finally, if $B^{2}-4 A C<0$, then there exists just an asymptotic stable equilibrium centre $\mathbf{W}$.

The case of three equilibrium centres is shown in figure 1 with $r_{g}=1 \cdot 5, r_{w}=1, s=$ $0.3, a=0.03, c_{g g}=0.7, c_{w g}=1, c_{g w}=2$, and $c_{w w}=1.03$, respectively. These are the same values for the system parameters used by Ludwig et al (1997). The stable equilibrium centre $\mathbf{W}$ corresponds to the extinction of grass, while the stable equilibrium centre $\mathbf{G}$ corresponds nearly to the opposite; namely, the grass density is high. Depending on the initial conditions, the system precisely approaches one of the stable equilibrium centres. As shown in figure 1, a trajectory starting from an initial state of $g=0.1$ and $w=0.1$ approaches the stable centre $\mathbf{G}$, while one ends up at the stable centre $\mathbf{W}$ if it begins from $g=0.1$ and $w=0 \cdot 3$. A separatrix passes through centre $\mathbf{S}$, and divides the whole permissible domain into two regions of attraction.

While equation set (1) catches some basic characteristics of a grazing system, it fails to account for variations in a real ecological environment. According to the model, the system would eventually approach either state $\mathbf{S}$ or state $\mathbf{W}$, and the outcome would depend entirely on the initial populations of the grass and woody vegetation respectively. 


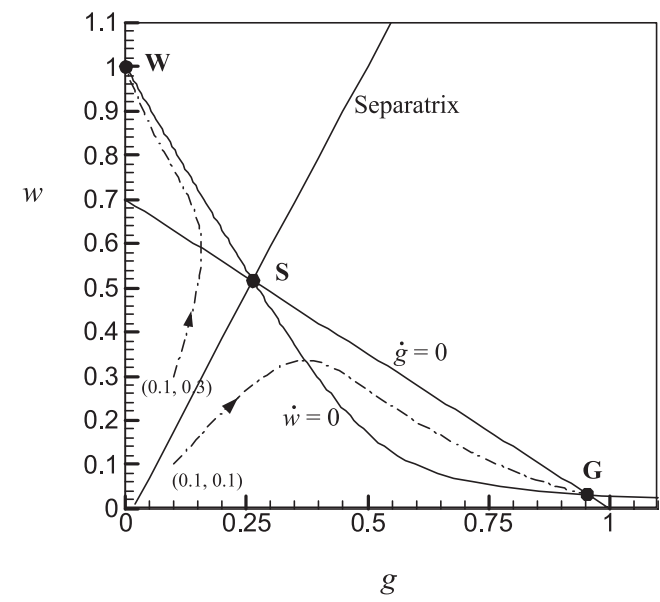

Figure 1. Phase plane of deterministic system (1).

\section{Randomized cattle-stocking rate}

The present paper is aimed at shedding some light on what happens if certain parameters in the system are allowed to change. Specifically, a stochastic model is investigated in which the stocking rate varies randomly with time. A randomized sinusoidal noise is used to model the variation part of the stocking rate. The stocking rate $s$ in equation set (1) is now replaced by

$$
s=s_{0}+s_{r} \xi(t), \quad s_{0}>s_{r}>0,
$$

where $s_{0}$ and $s_{r}$ are constants, and $\xi(t)$ is a randomized sinusoidal process described as follows,

$$
\xi(t)=\cos \theta(t), \quad \mathrm{d} \theta=v \mathrm{~d} t+\sigma \mathrm{d} B(t),
$$

in which $v$ is a positive constant, representing the mean frequency of the noise, $B(t)$ is a unit Brownian process (also known as the Wiener process), and $\sigma$ is also a positive constant adjusted according to the level of randomness of the noise. Equation set (6) indicates that $\xi(t)$ has a unit amplitude and a random phase. This randomized sinusoidal process was proposed independently by Dimentberg (1988) and Wedig (1989), and it has been used in the investigations of a variety of engineering problems (e.g. Dimentberg 1991, 1992, Li \& Lin 1995).

Figure 2 shows three spectral densities of the random process $\xi(t)$ computed for the cases of $v=1$ and three different values of $\sigma$, while figure 3 depicts those obtained for the cases of $\sigma=0.5$ and three different values of $\nu$. It is seen that equation set (6) can be used to model a variety of random phenomena. It reduces to a pure sinusoidal when $\sigma=0$. Parameter $v$ gives the peak location of the spectral density, which has a narrow peak when $\sigma$ is small. As $\sigma$ increases, the spectral density becomes increasingly flat. There are several advantages of using the randomized sinusoidal noise. It is bounded, and hence, more realistic than the unbounded white noise. It can never be periodic, except for the special case $\sigma=0$. Indeed, the model is versatile and realistic. 


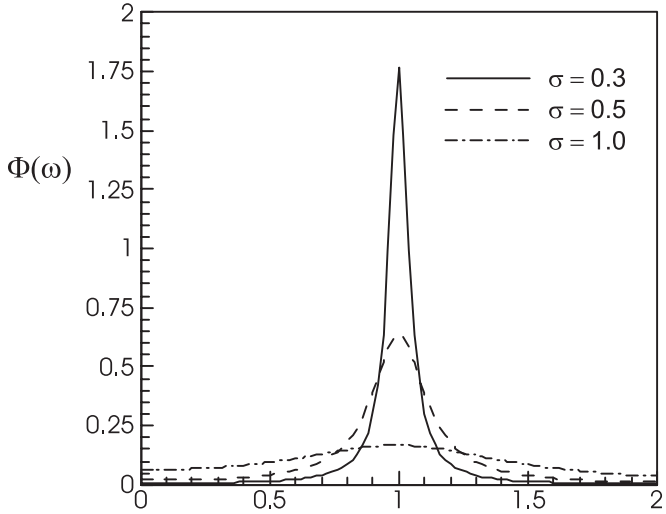

$\omega$
Figure 2. Spectral density of $\xi(t)$ for $v=1$ and different $\sigma$ values.

\section{Stochastic model for grazing system}

The randomized version of system (1) is given by:

$$
\begin{aligned}
\frac{\mathrm{d}}{\mathrm{d} t} G & =r_{g} G\left(1-s_{0}-c_{g g} G-c_{w g} W\right)+s_{r} r_{g} G \xi(t), \\
\frac{\mathrm{d}}{\mathrm{d} t} W & =r_{w}\left[a+W\left(1-c_{g w} G-c_{w w} W\right)\right],
\end{aligned}
$$

where $G(t)$ and $W(t)$ are now stochastic processes representing the uncertain grass and woody vegetation densities, respectively. A general comment on randomized cattle-stocking rate $S=s_{0}+s_{r} \xi(t)$ is in order. As indicated in the paper by Ludwig et al (1997), the cattlestocking rate generally varies slowly, and, in fact, this rate was represented by a constant in their paper. The question then arises as whether it is reasonable to use a stochastic process representation for this rate. We believe that our choice is indeed reasonable, because (a) the time frame of interest of the system is measured in multiple years, not days or months, in

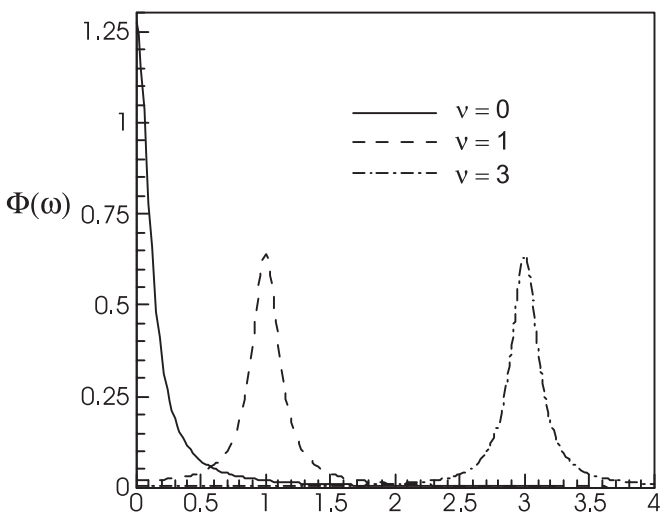

$\omega$
Figure 3. Spectral density of $\xi(t)$ for $\sigma=0.5$ and different $v$ values. 


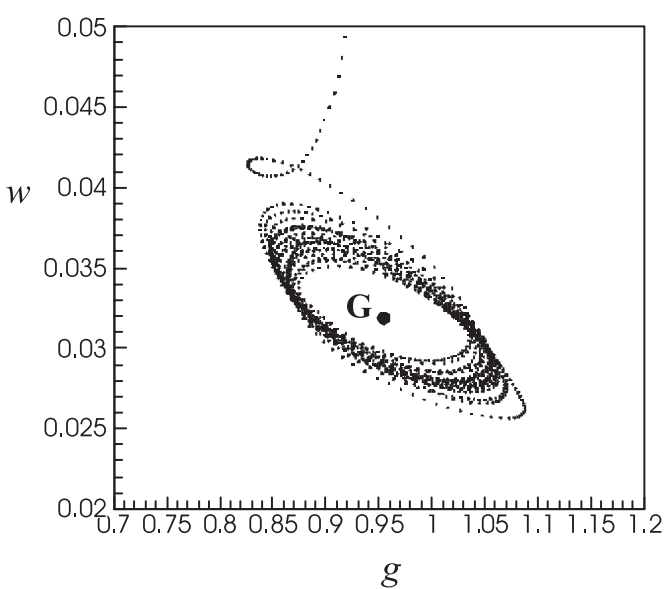

Figure 4. A trajectory of stochastic system (7).

which variations can be significant, and (b) the randomized sinusoidal is most versatile, and it can be used to model either fast or slow variations.

Analytical solution is presently not available for (7); thus, a Monte Carlo-type simulation study has been carried out with the same parameters as those in figure 1, i.e. $r_{g}=1 \cdot 5, r_{w}=$ $1, a=0.03, c_{g g}=0.7, c_{w g}=1, c_{g w}=2$, and $c_{w w}=1.03$. Figure 4 shows a trajectory of the stochastic system (7), with $s_{0}=0 \cdot 3, s_{r}=0 \cdot 1, v=1, \sigma=0.3$ beginning from $G(0)=0 \cdot 1$ and $W(0)=0 \cdot 1$. The starting point $(0 \cdot 1,01)$ of the trajectory is not included in the figure to permit using a larger scale, and thus a clearer display of the trajectory near point $\mathbf{G}$. It is seen that the steady state of the system can no longer be represented by a single point; instead, it is represented by a region around $\mathbf{G}$, which is in better agreement with what is expected of a real ecosystem.

When the cattle-stocking rate varies randomly with time, a distinctive separatrix of the system no longer exists, nor does an explicit boundary between two domains of attraction, as in the case of a deterministic system. Figure 5 shows that two identical systems can move

$w$

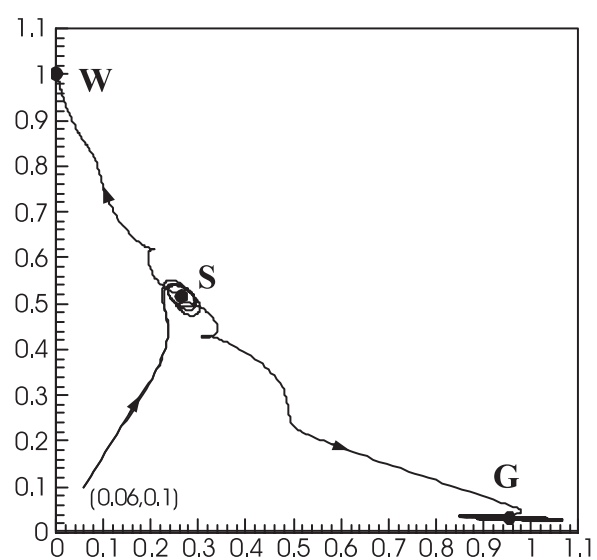

$g$
Figure 5. Two possible trajectories of stochastic system (7) beginning from the same point. 


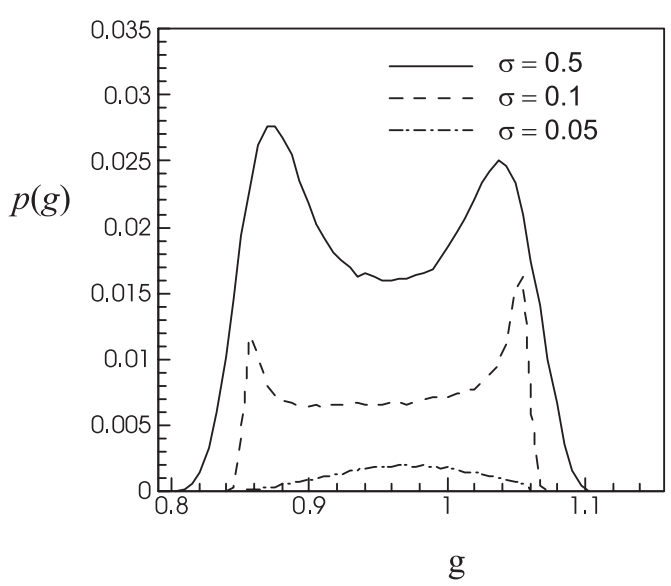

Figure 6. Probability density of grass density, computed with $v=1$, initial point $(0 \cdot 060,0 \cdot 1)$, and different $\sigma$ values.

along different paths beginning from the same initial state of $G(0)=0.06$ and $W(0)=0 \cdot 1$. Therefore, management and control are important to lead the system to a desired steady state.

Although the outcome for a single trajectory of system (7) is not predictable, the probability distribution of all possible outcomes can be determined. Figures 6 and 7 show the stationary probability density $p(g)$ of the grass density for the same system but for different initial states, where $g$ is the state variable of the stochastic process $G(t)$. In figure 6 , the initial point $(0.06,0.1)$ is in the attraction zone of stable state $\mathbf{W}$ of the deterministic model. In the stochastic case, however, it is not certain that the system ends up around state $\mathbf{W}$, and for the three cases of $\sigma=0.05,0.1$ and 0.5 , the probabilities of ending up around $\mathbf{W}$ are $0.975,0.829$ and 0.512 respectively. In other words, there are also probabilities that the system will ends up around state $\mathbf{G}$. Figure 7 shows the case of an initial state of (0.062, $0 \cdot 1)$ which is within the attraction zone of state $\mathbf{G}$. For the three cases of $\sigma=0.05,0 \cdot 1$ and 0.5 respectively, the stochastic system approaches its stationary state around point $\mathbf{G}$ with probabilities of $0.894,0.742$ and 0.620 respectively. It is interesting to note that the

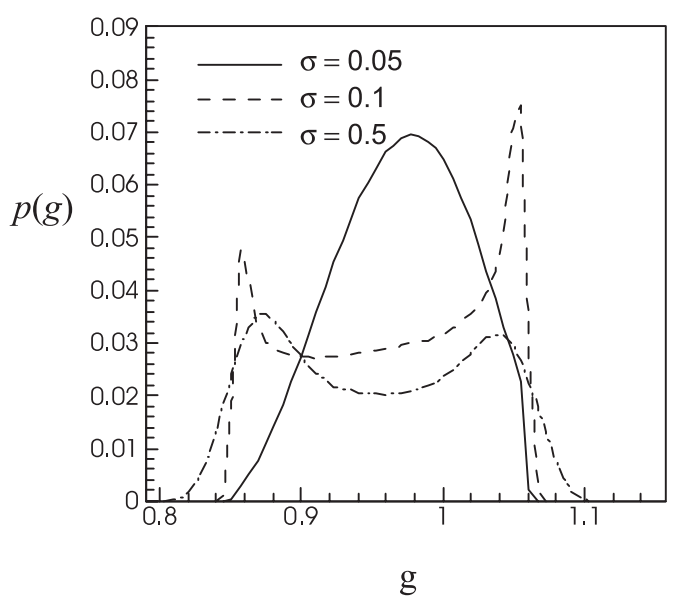

Figure 7. Probability density of grass density, computed with $v=1$, initial point $(0 \cdot 062,0 \cdot 1)$, and different $\sigma$ values. 
(a)

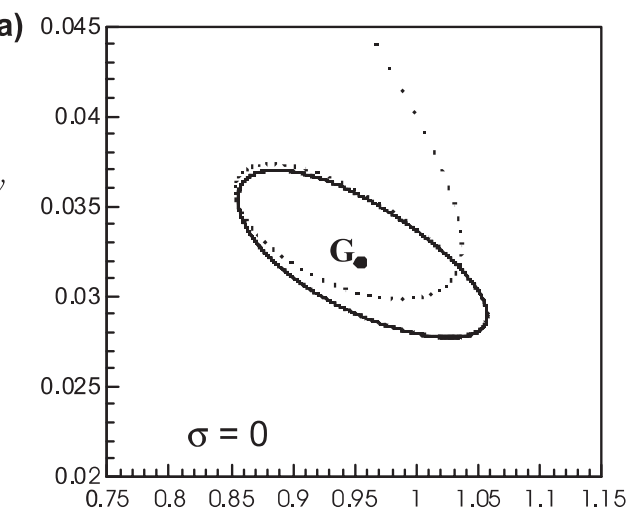

(c)

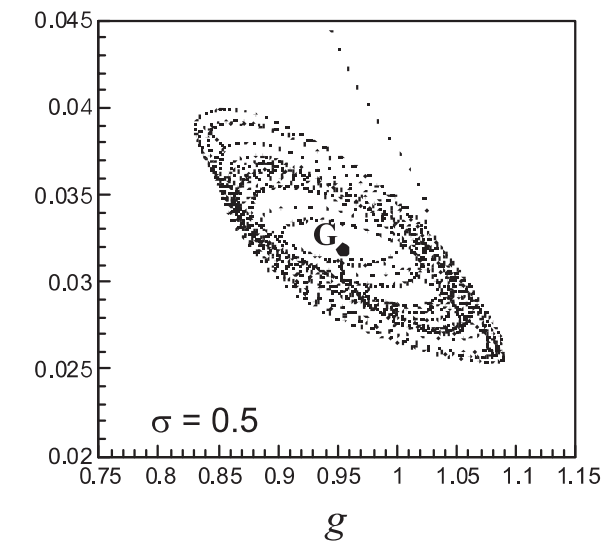

(b)

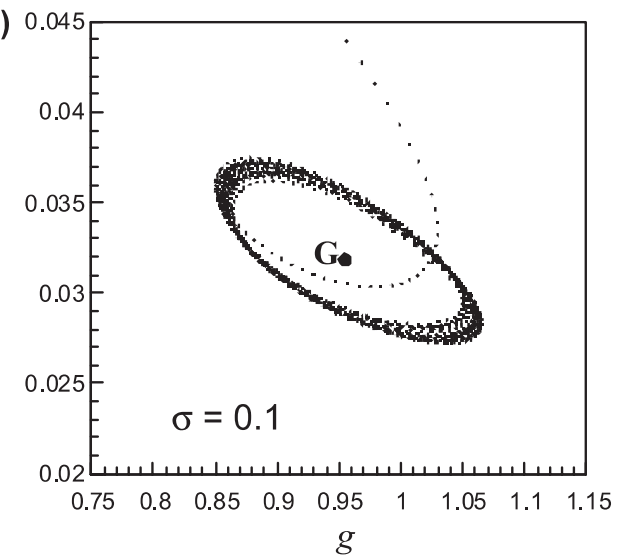

Figure 8. Trajectories computed for different randomness parameters, $\sigma=0$ (a), 0.1 (b) and $0.5(\mathbf{c})$.

system steady state depends not only on the system parameters, but also on the initial state. Furthermore, with a random variation in the cattle-stocking rate, which affects the grass consumption, the stable equilibrium point $\mathbf{G}$ is transformed into a region of stable steady states, while $\mathbf{W}$ remains a single stable equilibrium point. If random variation is also included in the source term of the woody vegetation, then point $\mathbf{W}$ is also diffused into a region of stable steady states.

\section{Effect of randomized stocking rate}

\subsection{Effect of randomness intensity $\sigma$}

As shown in equation set (6), parameter $\sigma$ measures the degree of randomness in the cattlestocking rate. Figure set 8 shows the trajectories of system (7) with $s_{0}=0 \cdot 3, s_{r}=0 \cdot 1, v=1$ for three different randomness parameters $\sigma=0,0.1$ and 0.5 respectively. For all three cases, the initial state is $G(0)=0 \cdot 1$ and $W(0)=0 \cdot 1$, which is outside the area shown in the figures. The case of $\sigma=0$ corresponds to a deterministic periodicity, and the steady-state trajectory is closed and is also periodic. For a nonzero $\sigma$, the steady-state trajectory spreads around this closed periodic curve. A larger randomness parameter $\sigma$ results in a greater spread of the steady state trajectories. 

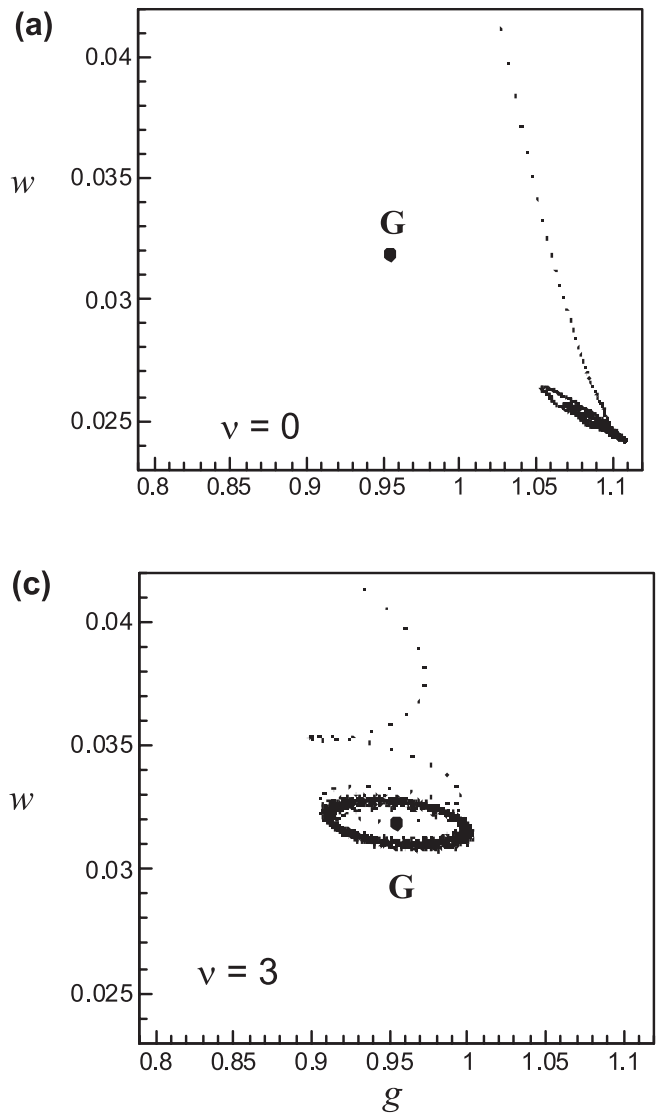

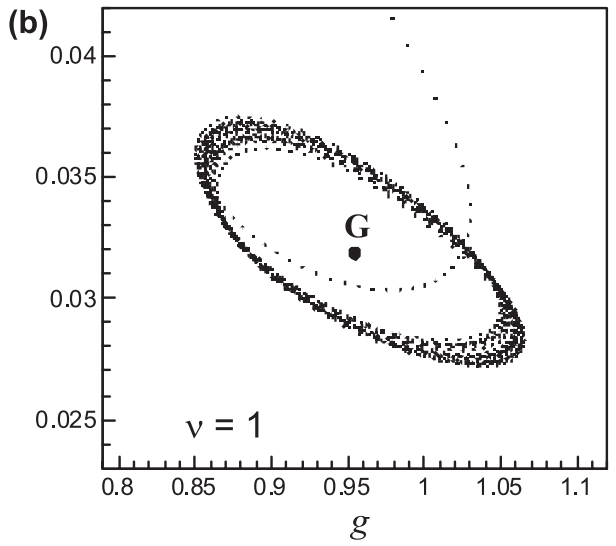

Figure 9. Trajectories computed for different randomness parameters, $v=0(\mathbf{a}), 1$ (b) and $3(\mathbf{c})$.

\subsection{Effect of mean frequency $v$}

Shown in figure set 9 are trajectories, beginning also from $g=0 \cdot 1$ and $w=0 \cdot 1$, and obtained for the case of $s_{0}=0 \cdot 3, s_{r}=0 \cdot 1, \sigma=0 \cdot 1$, and for three different mean frequencies of $v=0,1$ and 3 respectively. In the case of $v=0$, the variation part of the cattle-stocking rate is totally random, and the steady state is distributed in a region quite far from point $\mathbf{G}$. In the other two cases, $v=1$ and $v=3$, some "pseudo-periodicity" is implied in the cattle-stocking rate. The steady state regions are centred about $\mathbf{G}$, and a larger $v$ results in a smaller spread around $\mathbf{G}$.

\subsection{Effect of random amplitudes}

Shown in figure set 10 are trajectories, beginning again from $G(0)=0 \cdot 1$ and $W(0)=0 \cdot 1$, and obtained with $s_{0}=0 \cdot 3, v=1$ and $\sigma=0 \cdot 1$, and with three different noise amplitudes $s_{r}=1,2$ and 3. A larger $s_{r}$ indicates a larger amplitude of random variation of the cattlestocking rate, hence, resulting in a larger distribution region of the steady state.

\section{Conclusions}

Uncertain variabilities are always present in natural ecosystems, which must be accounted for in their mathematical models and in the formulation of effective management policies. The 
(a)
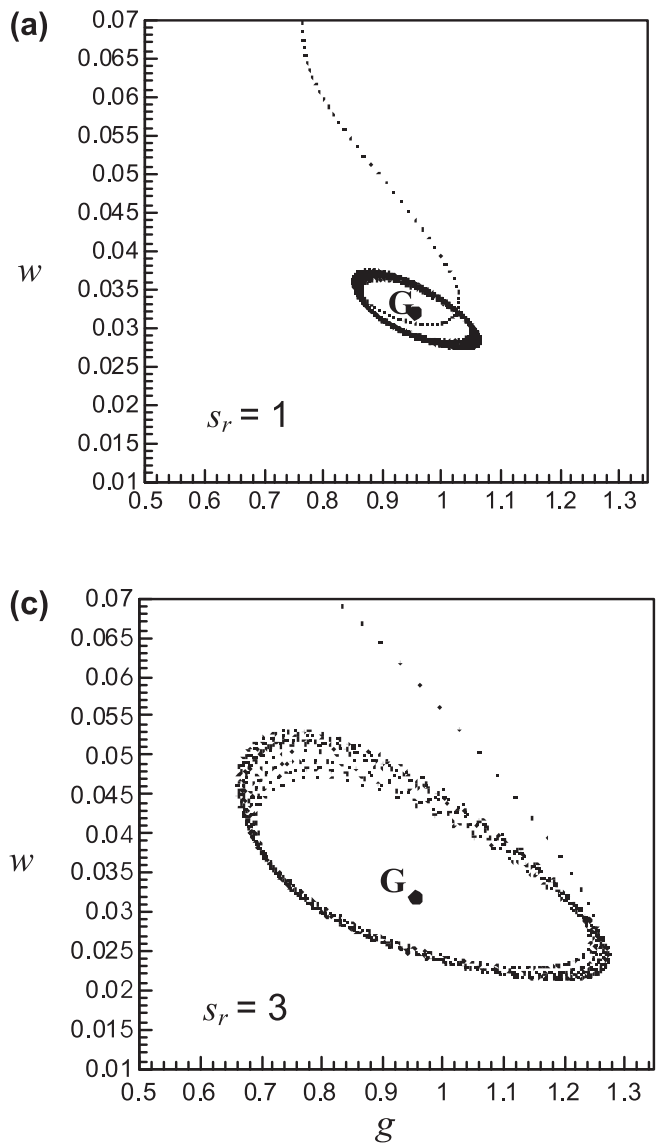

(b)

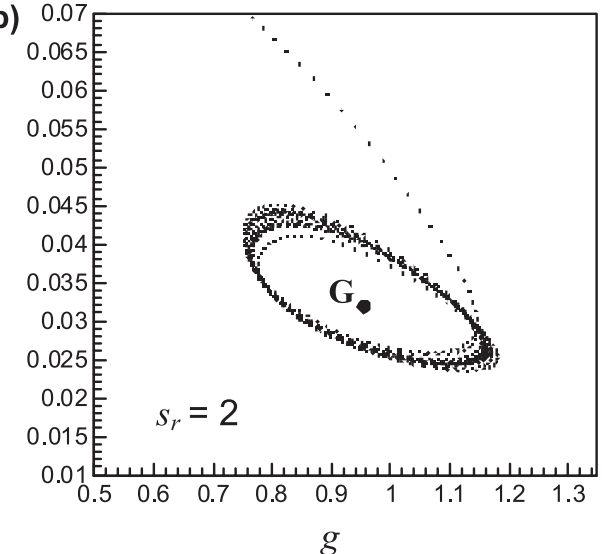

Figure 10. Trajectories computed for different randomness amplitudes $s_{r}=1(\mathbf{a}), 2$ (b) and 3 (c).

present investigation is an attempt in this direction, using the example of a semi-arid savanna grazing system. In particular, the stocking rate is assumed to be a constant plus a random variation. It is found that some qualitative changes in the system behaviours take place as a result. A single stable state of the deterministic system is expanded into a stable region, and the same initial state may lead to multiple final states.

In the present investigation, only one system parameter is treated as randomly varying with time. Similar approaches may apply to other system parameters, or for other ecosystems. The statistical properties of the random parameters of interest must be estimated from the observation data, which is, of course, time-consuming. Theoretical investigations are important to find out what types of data are most critically needed and what are not.

\section{References}

Dimentberg M F 1988 Statistical dynamics of nonlinear and time-varying systems (New York: Wiley) Dimentberg M F 1991 A stochastic model of parametric excitation of a straight pipe due to slug flow of a two-phase fluid. Proc. 5th Int. Conf. Flow-induced Vibrations, Brighton, pp. 207-209

Dimentberg M F 1992 Stability and subcritical dynamics of structures with spatially disordered parametric excitation. Probab. Eng. Mech. 7: 131-134 
Li Q C, Lin Y K 1995 New stochastic theory for bridge stability in turbulent flow, II. J. Eng. Mech. 121: $102-116$

Lotka A J 1925 Elements of physical biology (Baltimore, NJ: William and Wilkins)

Ludwig D, Jones D, Holling C S 1978 Qualitative analysis of insect outbreak systems: the spruce budworm and the forest. J. Animal Ecol. 47: 315-332

Ludwig D, Walker B, Holling C S 1997 Sustainability, stability, and resilience. Conserv. Ecol. 1: Article 7

Volterra V 1926 Variaziomi a flucttuazioni del numero d'individui in specie d'animali conviventi. Mem. Acad. Lincei 2: 31-113

Walker B, Ludwig D, Holling C S, Peterman R M 1981 Stability of semiarid savanna grazing systems. J. Ecol. 69: 473-498

Wedig W V 1989 Analysis and simulation of nonlinear stochastic systems. Nonlinear dynamics in engineering systems (ed.) W Schiehlen (Berlin: Springer-Verlag) pp. 337-344 